\title{
Folding Analytical Devices for Electrochemical ELISA in Hydrophobic R H Paper
}

\section{Citation}

Glavan, Ana C., Dionysios C. Christodouleas, Bobak Mosadegh, Hai Dong Yu, Barbara S. Smith, Joshua Lessing, M. Teresa Fernández-Abedul, and George M. Whitesides. 2014. " Folding Analytical Devices for Electrochemical ELISA in Hydrophobic R H Paper ." Analytical Chemistry 86 (24) (December 16): 11999-12007. doi:10.1021/ac5020782.

\section{Published Version}

doi:10.1021/ac5020782

\section{Permanent link}

http://nrs.harvard.edu/urn-3:HUL.InstRepos:16922668

\section{Terms of Use}

This article was downloaded from Harvard University's DASH repository, and is made available under the terms and conditions applicable to Open Access Policy Articles, as set forth at http:// nrs.harvard.edu/urn-3:HUL.InstRepos:dash.current.terms-of-use\#OAP

\section{Share Your Story}

The Harvard community has made this article openly available.

Please share how this access benefits you. Submit a story.

\section{Accessibility}




\title{
Folding Analytical Devices for Electrochemical ELISA
}

\section{in Hydrophobic $\mathbf{R}^{\mathbf{H}}$ Paper}

\author{
Ana C. Glavan ${ }^{1}$, Dionysios C. Christodouleas ${ }^{1}$, Bobak Mosadegh ${ }^{1}$, Hai Dong Yu ${ }^{1}$, Barbara \\ Smith $^{1}$, Joshua Lessing ${ }^{1}$, M. Teresa Fernández-Abedul ${ }^{1,3^{*}}$ and George M. Whitesides ${ }^{1,2^{*}}$ \\ ${ }^{1}$ Department of Chemistry and Chemical Biology, Harvard University, Cambridge MA \\ ${ }^{2}$ Wyss Institute for Biologically Inspired Engineering, Harvard University, Cambridge, MA \\ ${ }^{3}$ Departamento de Química Física y Analítica, Universidad de Oviedo, Spain
}

(*) Authors to whom correspondence should be addressed:

mtfernandeza@uniovi.es

gwhitesides@gmwgroup.harvard.edu

Target Journal: Anal. Chem. 


\begin{abstract}
This work describes a device for electrochemical ELISA designed for low-resource settings and diagnostics at the point of care. The device is fabricated entirely in hydrophobic paper, produced by silanization of paper with decyl trichlorosilane, and comprises two zones separated by a central axis: an embossed microwell, on the surface of which the antigen immobilization and recognition events occur, and a detection zone where the electrodes are printed. The two zones are brought in contact by folding the device along the central axis; the analytical signal is recorded from this folded configuration. The versatility of this device is shown through two proof-of-concept applications: an electrochemical direct ELISA for the detection of rabbit IgG as a model antigen in buffer, and an electrochemical sandwich ELISA for the detection of malarial histidine-rich protein ( $P f \mathrm{HRP} 2)$ from $P$. falciparum in spiked human serum. The limit of detection of the electrochemical sandwich ELISA for the quantification of $P f$ HRP2 in spiked human serum was $\sim 4 \mathrm{ng} / \mathrm{mL}(\sim 102 \mathrm{pM})$ — a value within the range of clinical relevant concentrations.
\end{abstract}


The enzyme-linked immunosorbent assay (ELISA) ${ }^{1}$ is a widely used method for detecting and quantifying biomarkers. The widespread utilization of ELISA in clinical laboratories lies in the specificity — based on the appropriate antibodies — of this type of assay, and on its high sensitivity—reflecting both the binding constant of the antibody and the enzymatic amplification step. ELISA has not so far achieved significant adoption outside clinical laboratories, in part because it requires cumbersome and expensive instrumentation (usually a microplate reader) to perform the readout, and skilled personnel to perform a laborious and time-consuming procedure. Reliable, inexpensive, and easy-to-use devices for electrochemical ELISA that can be used at the point of care - in the field, in a patient's home, or in a doctor's office—would be highly desirable, but are still not commercially available.

The present manuscript describes a proof-of-concept prototype for a low-cost, easy-tofabricate, portable and sensitive device for performing electrochemical ELISA. This device is constructed in cellulose paper that has been rendered hydrophobic by vapor-phase silanization with decyl trichlorosilane (" $\mathrm{C}_{10}{ }^{\mathrm{H}}$ paper"). ${ }^{2}$ We take advantage of the flexibility of this engineered paper to create microtiter wells by embossing; of its low surface free-energy to immobilize the capture biomolecules -antibodies and antigens - and to deposit carbon electrodes with high reproducibility on the surface of this paper; and of its foldability to "form" the electrochemical cell in which the solution of the sample contacts the electrodes. We show that this device can be used to perform direct and sandwich ELISAs able to detect picomolar or nanomolar concentrations of antigen.

Several different detection methods can be used to detect the products of the enzymatic amplification step of an ELISA, including among others, ${ }^{3,4}$ optical (colorimetric ELISA) or 
electrochemical (electrochemical ELISA) techniques.

In colorimetric ELISA, readouts are prone to errors caused by lighting conditions, sample color and turbidity, ${ }^{5}$ and are subjected to bias in color perception when recorded by the human eye. ${ }^{6}$ Spectrophotometers (usually microtiter plate readers, which cost more than $\$ 20,000$ ) can be used to overcome the subjectivity in visual readouts, but the increased cost and complexity of the instrumentation restrict the use of ELISA to well-equipped laboratories. Another drawback of conventional colorimetric ELISA is the consumption of relatively large volumes of reagents (50$360 \mu \mathrm{L}$ per well of a standard plastic 96 -well plate) required to attain sufficient optical path length for detection. The large volumes of reagents increase the cost of the materials used in the assay, as well as the time (several hours to overnight) required for completion of the assay. This time can be long because, for a given number of particles, the duration of each separate incubation and washing step reflects the distances over which reagents must diffuse. ${ }^{4}$ These limitations prevent the use of colorimetric ELISA in many applications in resource-limited settings, and in applications (agriculture, food safety, or environment) where cost and speed are important.

In electrochemical ELISA, the current produced by the oxidation or reduction of an electroactive substance-either when the potential is held constant (amperometry, recording the current-time response) or when the potential is scanned (voltammetry, recording the currentpotential response)-constitutes the analytical signal. ${ }^{7-9}$ Electrochemical detection of the end product of the enzymatic amplification step offers a number of advantages over colorimetric assays: it is completely insensitive to lighting conditions and color interferences, ${ }^{7,9}$ it can be performed with minimal user-bias, ${ }^{8,9}$ and it generates an electrical signal that can be used directly for web-based processing. 
Electrochemical detection can be performed using smaller volumes of reagents than those required by optical assays, since only a very thin film of the solution containing an electroactive compound is required for the electrochemical process to take place on the surface of the electrode. Moreover, electrochemical detection requires relatively simple and inexpensive instrumentation, as electrons are measured directly; in optical readouts, photons are converted into electrons using a photomultiplier or a CCD. Glucometers, the largest single class of pointof-care devices, cost less than $\$ 20$ and are used daily by millions of people worldwide. ${ }^{10}$ Our group $^{11}$ and others ${ }^{12-15}$ have recently described miniaturized, portable electrochemical readers, with performance comparable to that of conventional potentiostats, that can be fabricated inexpensively and are suitable for use at the point of care. ${ }^{11}$ Highly portable potentiostats are also commercially available from several manufacturers. ${ }^{16-19}$

Different conductive materials (e.g., carbon, ${ }^{6,10,20,21}$ metals, ${ }^{6,20,22,23}$ conductive polymers $^{21,22,24}$ ) can be used for the fabrication of electrodes on dielectric substrates such as synthetic polymers, ${ }^{25-28}$ glass, ${ }^{29,30}$ silicon, ${ }^{31}$ ceramics,,${ }^{32,33}$ paper, ${ }^{34-37}$ and recently omniphobic $\mathrm{R}^{\mathrm{F}}$ paper. ${ }^{38}$ Advances in methods of fabrication (including thick-film technologies such as gravure ${ }^{39}$ screen and stencil printing, ${ }^{37,40}$ drawing with conductive ink-filled rollerball pens, ${ }^{41}$ and inkjet printing, ${ }^{5,23,38}$ or thin-film technologies such as chemical vapor deposition, ${ }^{42,43}$ or sputtering ${ }^{42,44}$ ) have increased the performance and stability of the electrodes, and also reduced their cost enough to render them disposable after a single use.

Electrochemical ELISA can be carried out in standard microtiter plates, with the enzymatic reaction products being transferred at the end to an electrochemical measuring cell to perform the readout. ${ }^{45}$ A number of more sophisticated devices designed specifically for electrochemical immunoassays have also been developed, ${ }^{46-51}$ but most of the resulting systems have utilized 
silicon, glass, or polymers (particularly polydimethylsiloxane, PDMS) as the materials used in their fabrication, and the familiar microfabrication techniques developed for microelectronic devices (e.g., photolithography, thin film deposition, etching, and bonding) for important steps in the fabrication of these devices. Heineman et al performed one of the first electrochemical immunoassays $^{52}$ in 1993, using small-volume voltammetric detection of the pAP enzymatically generated in the immunoassay using a small incubator coupled with an electrochemical cell comprising an interdigitated array (IDA) gold working electrode and square counter and pseudoreference electrodes. The electrodes were fabricated on a thermally oxidized silicon wafer by photolithography and dry etching. Aguilar et al. ${ }^{47}$ reported an immunoassay format in which a recessed microdisk (for capturing antigen) and a nanoband gold electrode (for detection) were contained in a microcavity. Malhotra et al. ${ }^{53}$ developed an immunoassay using single-walled carbon nanotube forests, which were assembled from a suspension on pyrolytic graphite disks coated with a thin iron oxide-Nafion layer. Grant et al. ${ }^{54}$ developed an electrochemical immunoassay in which capture antibodies were embedded into conductive polypyrrole films. The poor permeability of these polymeric materials, however, hindered the diffusion of electroactive products in and out of the polymer film, resulting in slow response and low sensitivity. Escamilla-Gómez et al. ${ }^{55}$ developed dual immunosensors based on screen-printed carbon electrodes nanostructured with gold nanoparticles for the simultaneous detection of two antigens (free and total prostate-specific antigen). Recently, screen printed electrochemical arrays that can be integrated with standard microtiter plates have become commercially available; the price of each such array is in exces of $\$ 100$.

In most electrochemical immunoassays, the layers of immobilized antigens and antibodies are attached to the surface of the electrochemical transducers. ${ }^{46,53,56-59}$ One consequence of this 
design is that the immobilization of assay species on the surface of the sensor and the sequential incubation and washing steps, may cause fouling of the electrodes and may impede the electron transfer; ${ }^{60,61}$ to limit this potential problem, spatial separation of the immunoreactor and electrochemical detector has been suitable in many cases. ${ }^{48,62}$ Recently, Bhimji et al. ${ }^{48}$ developed an electrochemical ELISA in which the capture and recognition of the antibody occur in close proximity to large, three-dimensional gold microelectrodes. The assay is highly sensitive, but still suffers from drawbacks associated with complex fabrication procedure (i.e. soft lithography, etching and electrodeposition used for the fabrication of the glass chip with the working electrode) and the requirement for an external $\mathrm{Ag} / \mathrm{AgCl}$ reference electrode and a platinum auxiliary electrode.

The analytical device we describe is fabricated entirely on hydrophobic paper and comprises two zones separated by a central axis: an embossed microwell, on the surface of which the antigen immobilization and recognition events occur, and a detection zone where the electrodes are printed; the two zones are brought into contact by folding the device along the central axis, at which point when the analytical signal is recorded. This approach of assembling a device by folding components into contact has also been exploited, by $\mathrm{Crooks}^{63-65}$ and others ${ }^{66}$, as a means to fabricate paper-based analytical devices. Here, the folding of the device is not just a part of the fabrication process but a step in the analytical assay that allows us to minimize the fouling of the electrodes and increase the sensitivity of the assay.

As a proof-of-concept demonstration, we used the device for an electrochemical direct ELISA $^{7}$ using rabbit IgG as a model antigen and alkaline phosphatase-labeled anti-rabbit IgG as a model antibody. We chose this pair of antigen/ antibody because it is well-characterized in conventional colorimetric ELISAs; this background information enabled us to compare results 
between the two systems reliably. Once the method was optimized, we used the device to perform a electrochemical direct sandwich ${ }^{9}$ ELISA for the quantification of malarial histidinerich protein from Plasmodium falciparum ( $P f$ HRP2) spiked into human serum. This manuscript focuses solely on the electrochemical aspects of a demonstration of principle, and is not intended to demonstrate a completely prototyped system (including methods for sample preparation and addition).

\section{EXPERIMENTAL SECTION}

Materials and Chemicals. Whatman \#1 Chr chromatography paper was purchased from GE Healthcare (NJ, USA). Decyl trichlorosilane was purchased from Gelest Inc (Morrisville, PA). p-aminophenyl phosphate was purchased from Gold Biotechnology (St. Louis, MO). All chemicals were used as received without further purification. Graphite paste (C10903P14 Carbon/Graphite Ink) was purchased from Gwent Electronic Materials Ltd. (Torfaen, UK). The Ercon N-160 solvent thinner was purchased from Ercon Inc. (Wareham, MA). Graphite ink was prepared by mixing Gwent Graphite paste and Ercon N-160 solvent thinner in a 55:45 ratio). Goat anti-rabbit IgG antibody (A3689) and rabbit IgG were purchased from Sigma Aldrich (St Louis, MO). Mouse monoclonal anti $P f$ HRP2 (IgG, ABMAL-0445) and mouse monoclonal anti Pf HRP2 (IgM, ABMAL-0444) were purchased from Arista Biologicals, $P f$ HRP2 (A3000) was purchased from from CTK Biotech). The antibody against $P f$ HRP2 (ABMAL-0445, Arista Biologicals) was conjugated with alkaline phosphatase using an EZ-Link maleimide-activated alkaline phosphatase kit (Pierce Biotechnology, Rockford, IL). The Craft Robo Pro electronic craft cutter (Graphtec Corporation, Tokyo, Japan) was obtained from Silhouette America Inc (Orem, UT). Absorbance and fluorescence measurements were performed using a microtiter 
plate reader (model SpectraMax M2, Molecular Devices). Electrochemical measurements were carried out using an electrochemical analyzer (model Autolab PGSTAT302N, Metrohm).

Design and fabrication of the device. A 48 "well plate", comprising 48 individual devices, was fabricated from a $20 \times 20 \mathrm{~cm}$ sheet of chromatography paper (Whatman\#1 Chr chromatography paper) (Figure S1). Paper was embossed and subsequently functionalized with decyl trichlorosilane; three electrodes were deposited adjacent to each embossed feature (Figure 1). Individual devices were cut from the sheet using scissors.

We used embossing to shape the sheet of paper into a microtiter plate. We designed the shapes of the molds (positive and negative) using SolidWorks (Dassault Systèmes, S. A., Vélizy, France) (Figure S2), and fabricated them from acrylonitrile butadiene styrene (ABS) using a 3D printer (Dimension 3D, Stratasys Inc.) (Figure S3). We wet a flat sheet of Whatman Chr 1 paper with ethanol using a spray bottle, in order to render paper easier to shape and then we placed it between the pair of molds and pressed it manually. The embossed paper was then removed and allowed to dry in air.

We functionalized paper using a silanization reaction conducted in a chamber with a volume of $0.01 \mathrm{~m}^{3}$ at a temperature set at $105^{\circ} \mathrm{C} .{ }^{2}$ The silanizing reagent, decyl trichlorosilane, was transferred into a glass vial and placed inside the chamber together with the samples. Each experiment typically required approximately $100 \mathrm{mg}$ of silane in $5 \mathrm{~mL}$ of anhydrous toluene. Decyl trichlorosilane was vaporized at $95{ }^{\circ} \mathrm{C}$ under reduced pressure ( $\left.\sim 30 \mathrm{mbar}, 0.03 \mathrm{~atm}\right)$ and allowed to react for 5 minutes. Diffusion inside the reaction chamber was sufficient for an even distribution of the organosilane within the chamber. We refer to the paper produced according to this protocol using decyl trichlorosilane as $\mathrm{C}_{10}{ }^{\mathrm{H}}$ paper. 
We printed graphite electrodes on the surface of hydrophobic paper using a pen-on-paper approach. The template of the three-electrode system-comprising a working, counter and pseudoreference electrode - was designed using Adobe Illustrator (Adobe Systems Inc, San Jose, USA). All three electrodes were printed using graphite ink in order to minimize cost and simplify the procedure. We used $960 \mu \mathrm{m}$ roller ball pens (Gelly Roll Metallic pen, Sakura, Inc.) to dispense the graphite ink. To clean the pens, the ink barrel is separated from the rollerball tip, the commercial ink was removed using compressed air followed by flushing with ethanol and sonication in a bath of distilled water. Graphite ink was loaded into the pen, and the ink was initially forced through the rollerball tip by applying a burst of compressed air to the back of the ink barrel. The pen was installed in the blade-set of the Craft Robo Pro Electronic Craft Cutter, and the electrodes were printed according to the digital file (Figure S4 depicts the process). Printed graphite features are allowed to dry at room temperature for a minimum of $1 \mathrm{~h}$ before being used in experiments.

Evaluation of individual steps in electrochemical ELISA. First, we tested the ability of $\mathrm{C}_{10}{ }^{\mathrm{H}}$ paper to adsorb antibodies and protein onto its surface. We used an anti-human IgG antibody conjugated with FITC (FITC anti-human IgG) and recorded the fluorescence of the protein immobilized on the surface of wells in $\mathrm{C}_{10}{ }^{\mathrm{H}}$ paper. A $100-\mu \mathrm{M}$ solution of FITC anti-human IgG $(50 \mu \mathrm{L})$ was added to wells $(\mathrm{n}=14)$, allowed to incubate for 20 minutes and removed. Half of the wells $(n=7)$ were washed three times with a solution of PBST ( $1 \mathrm{x}$ phosphate buffer saline with $0.05 \%$ Tween-20, $\mathrm{pH} 7.4)$, then imaged, while the other half of the wells $(\mathrm{n}=7)$ were imaged directly (without a washing step). The difference between the fluorescence intensity of the two set of samples shows the amount of protein passively adsorbed onto the microwell, as compared 
to the unbound protein that can be washed out (Figure S5).

We then tested the ability of a solution of a standard blocking solution (1x phosphate buffer saline with containing $1 \%$ bovine serum albumin (BSA)) to prevent the nonspecific adsorption of proteins on $\mathrm{C}_{10}{ }^{\mathrm{H}}$ paper. Wells $(\mathrm{n}=14)$ in a microplate were blocked by incubation for 10 minutes with the blocking solution; 14 additional wells were used as controls. Solutions of FITC antihuman IgG were added to seven blocked wells, incubated for 30 minutes, and the signal was compared to the background (the fluorescence from wells that have been only blocked).

To evaluate the performance of the printed electrodes, we used cyclic voltammetry $(\mathrm{CV})$. We performed this analysis using 4-amino phenol (pAP) at three different concentrations ( $1 \mathrm{mM}$, $100 \mu \mathrm{M}, 10 \mu \mathrm{M})$

Electrochemical Analysis. We performed cyclic voltammetry (CV) and square wave voltammetry (SWV) (Figure S6) using a commercial potentiostat (Autolab PGSTAT12, Metrohm). We connected the paper device to the potentiostat using a reusable magnetic PDMS holder (Figure S7). The same holder can be used to interface with the portable electrochemical reader developed by Nemiroski et al. ${ }^{11}$ The parameters used for SWV are: pulse amplitude of $0.10 \mathrm{~V}$, square wave frequency of $10 \mathrm{~Hz}$, and step height of $0.005 \mathrm{~V}$, for scanning the potential between $-0.3 \mathrm{~V}$ and $0.3 \mathrm{~V}$ vs. graphite.

Electrochemical direct ELISA for detection of Rabbit IgG. Initially, each well was washed three times with $100-\mu \mathrm{L}$ volumes of a solution of PBST (1x phosphate buffer saline with $0.05 \%$ Tween-20, $\mathrm{pH} 7.4)$. A drop $(10 \mu \mathrm{L})$ of a solution with a known concentration of rabbit $\operatorname{IgG}$, 
ranging between $0.167 \mathrm{pM}$ to $167 \mathrm{nM}$, was added to each embossed well, the well were covered with a removable adhesive plastic (PET/EVA/LDPE, Fellowes sheet) and allowed to incubate for $30 \mathrm{~min}$ at room temperature. The solution was the aspired from the wells with a pipette and the surface of the wells was rinsed with $50 \mu \mathrm{L}$ of PBS (pH 7.4). Next, the surface was blocked with $50 \mu \mathrm{L}$ of a $1 \%$ solution of BSA in PBST for $5 \mathrm{~min}$, at room temperature. The solution was removed, and the well was washed with $50 \mu \mathrm{L}$ of PBST. A volume of $50 \mu \mathrm{L}$ of goat anti-rabbit antibody conjugated with alkaline phosphatase (ALP) was added and incubated for 5 min at room temperature. The plate was washed three times with PBST. The enzymatic amplification was performed by adding $50 \mu \mathrm{L}$ of $5 \mathrm{mM}$-aminophenyl phosphate in a buffer solution (1x PBS buffer, supplemented with $10 \mathrm{mM} \mathrm{MgCl}_{2}, \mathrm{pH} 9.5$ ) at room temperature in each embossed well. After the enzymatic reaction was allowed to occur for $10 \mathrm{~min}$, the device was folded, and the electrodes were allowed to come into contact with the solution. The electrochemical signal of $p$ aminophenol produced was recorded using square wave voltammetry (SWV).

Electrochemical sandwich ELISA for detection of Pf HRP2. Each well was washed three times with $100 \mu \mathrm{L}$ of a solution of PBST (1x phosphate buffer saline with $0.05 \%$ Tween-20, $\mathrm{pH}$ 7.4). The wells were coated by incubation of $50 \mu \mathrm{L}$ per well of capture antibody (mouse monoclonal anti Pf HRP2 IgM) at a concentration of $10 \mu \mathrm{g} / \mathrm{mL}$ in PBST for $1 \mathrm{~h}$. The wells were washed three times with PBST and then blocked using $50 \mu \mathrm{L}$ of a $1 \%$ solution of BSA in PBST for $1 \mathrm{~h}$ at room temperature. The solutions of recombinant $P f$ HRP2 were prepared in a 10 wt. \% solution of human serum in PBST. The wells were incubated with $50 \mu \mathrm{L}$ per well of solutions of human serum spiked with known concentrations of $P f \mathrm{HRP} 2$, for $30 \mathrm{~min}$ at room temperature. The wells were then washed five times with $50 \mu \mathrm{L}$ PBST and incubated with $50 \mu \mathrm{L}$ per well of 
ALP-conjugated detection antibody $(1 \mu \mathrm{g} / \mathrm{mL}$ in PBS, $\mathrm{pH}$ 7.4, mouse monoclonal anti $P f$ HRP2 IgG-ALP). After incubation for $20 \mathrm{~min}$ at room temperature under agitation, the solution was aspirated and the plate was washed three times with PBST. Detection was completed by adding $50 \mu \mathrm{L}$ of $5 \mathrm{mM}$ pAPP in a buffer (1x PBS buffer, supplemented with $10 \mathrm{mM} \mathrm{MgCl}_{2}, \mathrm{pH} 9.5$ ) at room temperature in each well. After the enzymatic reaction was allowed to occur for $15 \mathrm{~min}$, the device was folded, the electrodes were allowed to come into contact with the solution, and the signal was read using SWV.

\section{RESULTS AND DISCUSSION SECTION}

Design and fabrication of the device. We have chosen paper as a substrate for the fabrication of the electroanalytical device because it is widely available, inexpensive, lightweight, flexible, can be easily printed with conductive materials, and embossed or folded into 3D configurations; it also does not break into sharps and can be disposed of easily by burning. The ease of modifying its surface chemistry allowed us to render the paper hydrophobic using a five-minute vaporphase treatment with decyl trichlorosilane $\left(\mathrm{C}_{10}{ }^{\mathrm{H}}\right) .{ }^{2}$ The modified paper can easily be printed with conductive inks ${ }^{38}$ and facilitates the physical absorption of biomolecules on its surface through hydrophobic interactions. The roughness of the paper afforded a large surface area for the adsorption of proteins on the surface of the functionalized paper. We took advantage of these properties to immobilize antigens and antibodies on the surface of wells in hydrophobic paper. Embossing was chosen to shape the paper into a microtiter microplate because it is simple, rapid and requires inexpensive equipment (i.e., reusable molds generated using a 3D printer). Embossing is also compatible with mass fabrication methods such as reel-to-reel processes. 
We produced linear arrays of electrodes (a graphite working electrode, a graphite counter electrode and a graphite pseudo-reference electrode) spaced $1.5 \mathrm{~mm}$ apart using a $960 \mu \mathrm{m}$ roller ball pen filled with colloidal graphite ink on $\mathrm{C}_{10}{ }^{\mathrm{H}}$ paper (Figure 1). The rollerball pen filled with conductive graphite ink, under the control of a craft plotter, can transfer an easily modifiable digital pattern onto a sheet of paper to generate printed electrodes within minutes. This printing technique is useful for rapid prototyping and mass customization of electronic devices because it does not require custom-patterned components, such as screens, stencils and masks. This pen-onpaper approach, first introduced by Russo et al, ${ }^{41}$ afforded us a high degree of reproducibility in the printing of the electrodes, a high degree of flexibility in modifying and optimizing the design of the electrodes, and a fast method of fabricating relatively large numbers of electrodes ( 10 min to print a file comprising 70 three-electrode cells) (Figure S4).

The design of the device is aimed to minimize the fouling of the electrode by compounds required for a typical ELISA. We have spatially separated the process involved in the capture of the antigen, and the detection of the electroactive enzymatic product. The electroanalytical device incorporates printed electrodes proximal to, but not in contact with, the embossed microwell on the surface of which the immobilization of the antigen and the subsequent recognition events occur. The flexibility and foldability of the paper substrates allows us to bring the electrodes into contact with the reaction mixture only when the electrochemical detection step was ready to be performed. After the addition and removal of the series of reagents required for the ELISA was completed, the device was folded along a central axis to bring the electrodes in contact with the solution of the electroactive product of the enzymatic reaction (Figure 2).

Evaluation of different steps of electrochemical ELISA. The simplest electrochemical direct 
ELISA comprises six steps: (i) immobilizing the antigen on the surface of the paper, (ii) blocking the surface of the paper to prevent the nonspecific adsorption of proteins on any free sites not occupied by the antigen; (iii) washing away unbound protein antigen or antibodies; (iv) incubating with an antibody conjugated to an enzyme capable of converting an electrochemically inert substrate into an electroactive species; (v) adding and incubating with the enzymatic substrate; and (vi) recording the electrochemical turnover of the enzymatic product.

We used a fluorescently-labeled protein, FITC-conjugated anti-human IgG, to test the ability of the $\mathrm{C}_{10}{ }^{\mathrm{H}}$ paper to adsorb antibodies and protein antigens on the surface, as well as the ability of bovine serum albumin (BSA) to block any remaining active sites. Measurements of the fluorescent signal in each well (Figure S5) showed that the washing step did not change the intensity of the signal, confirming the strong immobilization of the fluorescent antigen on the surface of the hydrophobic $\mathrm{C}_{10}{ }^{\mathrm{H}}$ paper. After using a solution of BSA to block the surface of the embossed well, incubation with the fluorescently labeled protein (FITC-conjugated anti-human $\operatorname{IgG})$, followed by washing, produced a fluorescent signal indistinguishable from the background. (Wells that were not blocked showed a significantly higher fluorescent signal). Thus, a solution of BSA can be used to block effectively non-specific adsorption of proteins on the surface of $\mathrm{C}_{10}{ }^{\mathrm{H}}$ hydrophobic paper. We also confirmed that a solution of BSA was able to effectively block non-specific protein binding: the blocked wells, after incubation with FITC-conjugated antihuman IgG, gave fluorescent signals indistinguishable from the background.

Generation of the electroactive species. There are several examples of enzyme/substrate pairs that can be used to generate electroactive products. Heineman et al. pioneered the use of 4-aminophenyl phosphate (pAPP) as an enzymatic substrate in electrochemical 
immunoassays. ${ }^{67,68}$ In this work, we have chosen alkaline phosphatase (ALP) as the enzyme and 4-aminophenyl phosphate (pAPP) as the substrate, because they generate an electrochemically active product, 4-aminophenol (PAP) that can be oxidized at a relatively low potential ( $\sim 100 \mathrm{mV}$ vs. graphite) to a quinone imine. As the noise in electrochemical detection typically increases with increasing potential, this low oxidation potential of pAP is advantageous. This low potential also makes the interference from the oxidation of other species in the sample unlikely. In addition, a wide variety of ALP-conjugated antibodies are commercially available.

Recording the analytical signal. Since wicking lowers the resolution with which inks can be patterned on paper, ${ }^{38}$ the relatively low surface free energy of the hydrophobic paper $\left(\theta_{\text {app }}{ }^{\mathrm{H} 2 \mathrm{O}}>130^{\circ}\right)^{2}$ allowed us to print electrodes with well-defined surface areas and relatively low variation in performance from device to device. To evaluate the performance of the electrodes over a potential window, we recorded the cyclic voltammetry $(\mathrm{CV})$ of 4-amino phenol $(\mathrm{pAP})$ at three different concentrations $(1 \mathrm{mM}, 100 \mu \mathrm{M}, 10 \mu \mathrm{M})$. To characterize the variation in the performance of the electrodes between different batches, we determined the anodic peak current, $\mathrm{i}_{\mathrm{p}}$, measured from the decaying cathodic current as a baseline. ${ }^{69}$ We observed very small deviceto-device variation in the performance of the electrodes, as indicated by the similar curves and the small relative standard deviation (RSD, defined as the percentage ratio of the standard deviation to the mean of the distribution) of $\sim 4.5-6.7 \%$ in the peak current, $i_{p}$, in the cyclic voltammograms of different electrodes fabricated in different batches $(n=7)$ (Figure 3).

Electrochemical direct ELISA for detection of Rabbit IgG. We used rabbit IgG as a model antigen $^{7}$ and anti-rabbit IgG-ALP conjugate as detection antibody to test the performance of our 
device in a direct ELISA. Rabbit $\operatorname{IgG}(10 \mu \mathrm{L})$ was applied to each well in tenfold dilutions ranging from $0.167 \mathrm{pM}$ to $167 \mathrm{nM}$, the well was covered and the solution was allowed to incubate for 30 minutes at room temperature. After washing with PBST and blocking the surface of the well with a solution of BSA, the anti-rabbit IgG-ALP conjugate was added to each well and bound with the rabbit IgG. After the excess was washed away, a solution of the electrochemical substrate pAPP was added to the microwells. The conversion of pAPP to the electrochemically active pAP was allowed to proceed for 10 minutes, before the device was folded along a central axis to bring the electrodes into contact with the solution of the electroactive enzymatic product, and the electrochemical signal was measured (Figure 4).

Cyclic voltammetry is not an ideal method for accurate quantitation of electroactive species, because the correction for the capacitive current is typically uncertain. ${ }^{69}$ In order to increase the sensitivity of our measurements, we used square-wave voltammetry (SWV)—a fast electrochemical technique developed by Osteryoung and Osteryoung. ${ }^{70}$

Square wave voltammetry allows rejection of background currents, including currents that are largely independent of potential, currents arising from convective mass transport and capacitive currents generated by a time variation of the capacitance itself. These currents, typically large in voltammetry, are critical factors in determining the detection limits. Since these currents are relatively slow varying, SWV results in significant current cancellation.

Square wave voltammetry increases the ratio of Faradaic to capacitive current ${ }^{69}$ by using a symmetrical square wave on a staircase as a waveform (see Figure S6c). According to the Cottrell equation, the decay of the Faradaic current is inversely proportional to the square root of time, and proportional to the bulk concentration of the electroactive species, $\mathrm{c}\left(\mathrm{mol} \mathrm{cm}{ }^{-3}\right)$, the area of the electrode, $\mathrm{A}\left(\mathrm{cm}^{2}\right)$, the diffusion coefficient $\mathrm{D}\left(\mathrm{cm}^{2} \mathrm{~s}^{-1}\right)$, the number $\mathrm{n}$ of electrons 
transferred per molecule and Faraday's constant:

$$
i_{F}(t)=\frac{n F A D^{1 / 2} c}{(\pi t)^{1 / 2}}
$$

The decay of the double layer charging current is an exponential function of time t, the resistance $R_{u}(\Omega)$ of the solution and the double-layer capacitance $C_{d l}(F)$, and a linear function of the applied potential pulse $\Delta E(V)$ :

$$
i_{c}(t)=\frac{\Delta E}{R_{u}} e^{-t /\left(R_{u} C d l\right)}
$$

Thus the capacitive current decays exponentially with time, whereas the Faradaic current is a more slowly varying function of time, decaying with the inverse of the square root of time. ${ }^{71}$

Since the current is sampled at the end of each pulse, and the capacitive current in these experiments is negligible compared to the Faradaic current, the background is suppressed; this suppression lowers the limit of detection and increases the sensitivity. SWV allowed us to measure pAP at substantially lower concentrations than was possible with $\mathrm{CV}$. The parameters for SWV measurement were chosen in preliminary trials to provide the highest signal-to-noise ratio in the detection of a $100 \mathrm{nM}$ solution of pAP in PBS (data not shown); these parameters were chosen to be a pulse amplitude of $0.10 \mathrm{~V}$, square wave frequency of $10 \mathrm{~Hz}$, and a step height of $0.005 \mathrm{~V}$ for scanning the potential between $-0.3 \mathrm{~V}$ and $0.3 \mathrm{~V}$ vs. graphite. The measurements were performed using a commercial potentiostat; the parameters we used are, however, compatible with the portable electrochemical reader developed by Nemiroski et al. ${ }^{11}$

We generated a calibration curve of the peak current recorded by SWV versus the concentration of rabbit IgG at concentrations ranging from $0.167 \mathrm{pM}$ to $167 \mathrm{nM}$ (in PBS, $\mathrm{pH}$ 
7.4), as shown in Figure 4. The peak current of pAP was found to be proportional to the concentration of rabbit IgG. We can approximate the curve as linear in the concentration range of 10 and $1000 \mathrm{pM}$.

At the present state of development, the limit of detection (LOD, as defined by the concentration that results in a signal that is equal to the average signal of the control—at zero concentration of IgG — plus three times the standard deviation in the signal of the control) of our electrochemical direct ELISA for rabbit IgG was estimated to be $2 \mathrm{pM}$. The LOD was lower than that determined for conventional absorbance ELISA ( $55 \mathrm{pM}$ ) using the same antigen/antibody pair. The detection limit we reached using electrochemical ELISA was, however, higher than values reported for ELISA based on different detection technologies (chemiluminescence ${ }^{72}$, electrochemi-luminescence ${ }^{73}$ ) and different antigen/antibody pairs. For the detection of human cardiac Troponin $\mathrm{T}(\mathrm{cTnT})$, for example, chemiluminescence provides limits of detection as low as $0.15 \mathrm{pM}$, at a cost of $\sim \$ 15$ per assay. ${ }^{72}$ The decrease in detection limits comes at the expense of increased cost, and heavier and more cumbersome hardware.

Electrochemical sandwich ELISA for detection of Pf HRP2. We used our device to detect and quantify levels of $P f \mathrm{HRP} 2$ in spiked diluted human serum, using a sandwich ELISA assay. We immobilized the capture antibodies (mouse anti $P f \mathrm{HRP} 2$ IgM) on the hydrophobic $\mathrm{C}_{10}{ }^{\mathrm{H}}$ paper surface by passive adsorption. We used a concentration of capture antibody of $10 \mu \mathrm{g} / \mathrm{mL}$ in order to facilitate the adsorption of the antibody in a "Fab-up" orientation. ${ }^{74}$ We conjugated the detection antibody (mouse anti $P f \mathrm{HRP} 2 \mathrm{IgG}$ ) to ALP in order to minimize the number of incubation and washing steps in the assay.

We used human serum samples spiked with recombinant $P f \mathrm{HRP} 2$ at concentrations ranging 
from $1 \mathrm{pg} / \mathrm{mL}$ to $10^{6} \mathrm{pg} / \mathrm{mL}$ as the samples to be tested and we generated a calibration curve of the concentration of $P f \mathrm{HRP} 2$ protein vs the peak current of pAP recorded by SWV (Figure 5). The limit of detection (LOD; taken at three standard deviations above the mean of the blank measurement) was $\sim 4 \mathrm{ng} / \mathrm{mL}(\sim 102 \mathrm{pM})$. The LOD in a conventional colorimetric ELISA was $\sim 17.6 \mathrm{ng} / \mathrm{mL}$ for the same pair of antigen and antibody.

According to a study by Dondorp et al, the mean $(95 \% \mathrm{CI})$ concentration of $P f \mathrm{HRP} 2$ in the plasma of 337 patients with falciparum malaria was $0.84 \mu \mathrm{g} / \mathrm{ml}$, corresponding to a range of $0.57 \mu \mathrm{g} / \mathrm{mL}$ to $1.11 \mu \mathrm{g} / \mathrm{ml} .{ }^{75}$ This result suggests that our assay can detect the presence of Pf HRP2 in concentrations that are clinically relevant.

\section{CONCLUSION}

This study describes a device for electrochemical ELISA designed specifically for low-resource settings. Made entirely from hydrophobic paper and printed carbon electrodes, the device is versatile: it can be used for direct, indirect, or sandwich ELISA, and it can detect any analyte for which appropriate antibodies and antigens have been developed. Arrays of these devices can interface with multichannel electrochemical readers to perform multiplex analyses.

This device has four significant features: (i) it is quantitative and sensitive; (ii) it is cost effective ( $\sim \$ 0.005$ per device, not including the cost of the biochemical reagents), due both to the inexpensive materials and method used for its fabrication, and to its relatively low consumption of reagents (compared to conventional ELISA); (iii) it is easily stored and transported; (iv) contaminated devices can be easily disposed of after use (by incineration); (iv) the equipment used for electrochemical detection is lighter, smaller, and less expensive than conventional 
spectrophotometers or optical scanners. The paper-based device described here can be coupled with the miniaturized electrochemical readers with performance comparable to that of commercial potentiostats, as described previously by Nemirosky et al. ${ }^{11}$ The cost of these devices and the ease of interfacing with telephone and web-based systems for transferring information to medical and public health personnel, and for processing and storage, can transform the way electrochemical analysis is performed into the field.

This work, however, still does not meet all the demands of low-resource settings. The need to add and remove small, fixed volumes of reagents manually and sequentially still limits the practicality of this device in field applications. Combining other technologies, such as microfluidic systems, may automate the delivery of these reagents. These caveats notwithstanding, the work demonstrates the potential of this kind of device and analysis.

\section{ACKNOWLEDGEMENTS}

This work was funded in part by the Bill \& Melinda Gates Foundation under award 51308. M.T.Fernández-Abedul thanks the University of Oviedo (Campus de Excelencia Internacional) and the Spanish Ministry of Economy and Competitiveness (project MICINN CTQ2011-25814) for funding her stay at Harvard University. We thank Jeremy Schonhorn, Jason Rolland, and Christina Swanson from Diagnostics for All (Cambridge, MA) for helpful discussions. Supporting information is available online from Wiley InterScience or from the website of the Whitesides group (http://gmwgroup.harvard.edu/pubs). 


\section{REFERENCES}

(1) Crowther, J. In ELISA: Theory and Practice, Crowther, J., Ed.; Humana Press, 1995, pp 1-34.

(2) Glavan, A. C.; Martinez, R. V.; Subramaniam, A. B.; Yoon, H. J.; Nunes, R. M. D.; Lange,

H.; Thuo, M. M.; Whitesides., G. M. Adv. Funct. Mater. 2013 DOI: 10.1002/adfm.201300780.

(3) Engvall, E.; Perlmann, P. Immunochemistry 1971, 8, 871-\&.

(4) Ihalainen, P.; Pettersson, F.; Pesonen, M.; Viitala, T.; Maattanen, A.; Osterbacka, R.; Peltonen, J. Nanotechnology 2014, 25.

(5) Kwon, O.-S.; Kim, H.; Ko, H.; Lee, J.; Lee, B.; Jung, C.-H.; Choi, J.-H.; Shin, K. Carbon 2013, 58, 116-127.

(6) Glatzel, S.; Schnepp, Z.; Giordano, C. Angew. Chem. Int. Ed. 2013, 52, 2355-2358.

(7) Kissinger, P.; Heineman, W. R. Laboratory Techniques in Electroanalytical Chemistry, revised and expanded, Second ed.; Marcel Dekker, Inc.: New York, NY, 1996.

(8) pp According to the manufacturer's data sheet:

Ecoflex: http://www.smooth-on.com/index.php?cPath=1130.

Paper: https://http://www.vwrsp.com/catalog/product/index.cgi?catalog_number=21913-21211.

(9) Wang, J. Analytical electrochemistry; John Wiley \& Sons: Hoboken, New Jersey, 2006.

(10) Maxwell, E. J.; Mazzeo, A. D.; Whitesides, G. M. MRS Bull. 2013, 38, 309-314.

(11) Nemiroski, A.; Christodouleas, D.; Hennek, J.; Maxwell, E. J.; Fernandez-Abedul, M. T.;

Whitesides, G. M. Proc. Natl. Acad. Sci. U. S. A. 2014.

(12) Rowe, A. A.; Bonham, A. J.; White, R. J.; Zimmer, M. P.; Yadgar, R. J.; Hobza, T. M.; 
Honea, J. W.; Ben-Yaacov, I.; Plaxco, K. W. PLoS One 2011, 6, e23783.

(13) Lillehoj, P. B.; Huang, M.-C.; Truong, N.; Ho, C.-M. Lab Chip 2013, 13, 2950-2955.

(14) Huang, C.-Y.; Lin, H.-Y.; Wang, Y.-C.; Liao, W.-Y.; Chou, T.-C. In Proceedings at the 2004 IEEE Asia-Pacific Conference on Circuits and Systems; IEEE, 2004, pp 633-636.

(15) Blanco, J.; Ferrero, F.; Campo, J.; Antón, J.; Pingarrón, J.; Reviejo, A.; Manso, J. In Proceedings of the Instrumentation and Measurement Technology Conference, IMTC 2006. ; IEEE, 2006, pp 690-694.

(16) Dropsens. $\mu$ Stat 200 Bipotentiostat. Retrieved at:

http://www.dropsens.com/en/pdfs_productos/new_brochures/stat200.pdf, April 16, 2014.

(17) Dropsens. $\mu$ Stat 4000P Multipotentiostat. Retrieved at:

http://www.dropsens.com/en/pdfs_productos/new_brochures/stat4000p.pdf, April 16, 2014.

(18) Palmsens. EMStat. Retrieved at: http://www.palmsens.com/en/instruments/emstat/, April $16,2014$.

(19) Pine Research Instrumentation; WaveNano USB Potentiostat. Retrieved at: https://http://www.pineinst.com/echem/viewproductpage.asp?pageID=63\&productID=47292, April 16, 2014.

(20) Lee, H.-H.; Chou, K.-S.; Huang, K.-C. Nanotechnology 2005, 16, 2436.

(21) Wang, X.; Zhi, L.; Müllen, K. Nano Lett. 2008, 8, 323-327.

(22) Jonas, F.; Heywang, G. Electrochim. Acta 1994, 39, 1345-1347.

(23) Määttänen, A.; Ihalainen, P.; Pulkkinen, P.; Wang, S.; Tenhu, H.; Peltonen, J. ACS Appl. Mater. Inter. 2012, 4, 955-964. 
(24) Snook, G. A.; Kao, P.; Best, A. S. J. Power Sources 2011, 196, 1-12.

(25) Zhang, S.; Wright, G.; Yang, Y. Biosens. Bioelectron. 2000, 15, 273-282.

(26) McDonald, J. C.; Whitesides, G. M. Acc. Chem. Res. 2002, 35, 491-499.

(27) Walker, S. B.; Lewis, J. A. J. Am. Chem. Soc. 2012, 134, 1419-1421.

(28) Alligrant, T. M.; Nettleton, E. G.; Crooks, R. M. Lab Chip 2013, 13, 349-354.

(29) Goss, C. A.; Charych, D. H.; Majda, M. Anal. Chem. 1991, 63, 85-88.

(30) Gooding, J. J. Electrochim. Acta 2005, 50, 3049-3060.

(31) Kelley, S.; Deluga, G.; Smyrl, W. AIChE J. 2002, 48, 1071-1082.

(32) Burmeister, J. J.; Moxon, K.; Gerhardt, G. A. Anal. Chem. 2000, 72, 187-192.

(33) García-González, R.; Fernández-Abedul, M. T.; Pernía, A.; Costa-García, A. Electrochim. Acta 2008, 53, 3242-3249.

(34) Nie, Z.; Deiss, F.; Liu, X.; Akbulut, O.; Whitesides, G. M. Lab Chip 2010, 10, 3163-3169.

(35) Liu, H.; Xiang, Y.; Lu, Y.; Crooks, R. M. Angew. Chem. 2012, 124, 7031-7034.

(36) Apilux, A.; Dungchai, W.; Siangproh, W.; Praphairaksit, N.; Henry, C. S.; Chailapakul, O. Anal. Chem. 2010, 82, 1727-1732.

(37) Nie, Z.; Nijhuis, C. A.; Gong, J.; Chen, X.; Kumachev, A.; Martinez, A. W.; Narovlyansky, M.; Whitesides, G. M. Lab Chip 2010, 10, 477-483.

(38) Lessing, J.; Glavan, A. C.; Walker, S. B.; Keplinger, C.; Lewis, J. A.; Whitesides, G. M. Adv. Mater. 2014.

(39) Bagel, O.; L’Hostis, E.; Lagger, G.; Osborne, M. D.; Seddon, B. J.; Girault, H. H.; Brack, 
D.; Loyall, U.; Schäfer, H. J. Electroanal. Chem. 1999, 469, 189-195.

(40) Wang, J.; Tian, B.; Nascimento, V. B.; Angnes, L. Electrochim. Acta 1998, 43, 3459-3465.

(41) Russo, A.; Ahn, B. Y.; Adams, J. J.; Duoss, E. B.; Bernhard, J. T.; Lewis, J. A. Adv. Mater. 2011, 23, 3426-+.

(42) Foster, C. M.; Bai, G. R.; Csencsits, R.; Vetrone, J.; Jammy, R.; Wills, L. A.; Carr, E.; Amano, J. J. Appl. Phys. 1997, 81, 2349-2357.

(43) Hecht, D. S.; Hu, L. B.; Irvin, G. Adv. Mater. 2011, 23, 1482-1513.

(44) Nanto, H.; Minami, T.; Shooji, S.; Takata, S. J. Appl. Phys. 1984, 55, 1029-1034.

(45) Sha, H.; Bai, Y.; Li, S.; Wang, X.; Yin, Y. Am. J. Orthod. Dentofacial Orthop. 2014, 145, $36-40$.

(46) Dequaire, M.; Degrand, C.; Limoges, B. Anal. Chem. 2000, 72, 5521-5528.

(47) Aguilar, Z. P.; Vandaveer, W. R.; Fritsch, I. Anal. Chem. 2002, 74, 3321-3329.

(48) Bhimji, A.; Zaragoza, A. A.; Live, L. S.; Kelley, S. O. Anal. Chem. 2013, 85, 6813-6819.

(49) Pampalakis, G.; Kelley, S. O. Analyst 2009, 134, 447-449.

(50) Rossier, J. S.; Girault, H. H. Lab Chip 2001, 1, 153-157.

(51) Tang, D. P.; Su, B. L.; Tang, J.; Ren, J. J.; Chen, G. N. Anal. Chem. 2010, 82, 1527-1534.

(52) Niwa, O.; Xu, Y.; Halsall, H. B.; Heineman, W. R. Anal. Chem. 1993, 65, 1559-1563.

(53) Malhotra, R.; Patel, V.; Vaque, J. P.; Gutkind, J. S.; Rusling, J. F. Anal. Chem. 2010, 82, 3118-3123.

(54) Grant, S.; Davis, F.; Pritchard, J. A.; Law, K. A.; Higson, S. P. J.; Gibson, T. D. Anal. Chim. 
Acta 2003, 495, 21-32.

(55) Escamilla-Gomez, V.; Hernandez-Santos, D.; Gonzaez-Garcia, M. B.; Pingarron-Carrazon, J. M.; Costa-Garcia, A. Biosens. Bioelectron. 2009, 24, 2678-2683.

(56) Bange, A.; Halsall, H. B.; Heineman, W. R. Biosens. Bioelectron. 2005, 20, 2488-2503.

(57) Luppa, P. B.; Sokoll, L. J.; Chan, D. W. Clin. Chim. Acta 2001, 314, 1-26.

(58) Niwa, O.; Xu, Y.; Halsall, H. B.; Heineman, W. R. Anal. Chem. 1993, 65, 1559-1563.

(59) Wang, J. Biosens. Bioelectron. 2006, 21, 1887-1892.

(60) Ko, H.; Lee, J.; Kim, Y.; Lee, B.; Jung, C.-H.; Choi, J.-H.; Kwon, O.-S.; Shin, K. Adv. Mater. 2014, n/a-n/a.

(61) In Electroanalytical Methods: Guide to Experiments and Applications, Scholz, F., Ed.; Springer Verlag Heidelberg, 2002.

(62) Abad-Villar, E. M.; Fernandez-Abedul, M. T.; Costa-Garcia, A. Biosens. Bioelectron. 2002, $17,797-802$.

(63) Liu, H.; Li, X.; Crooks, R. M. Anal. Chem. 2013, 85, 4263-4267.

(64) Scida, K.; Li, B. L.; Ellington, A. D.; Crooks, R. M. Anal. Chem. 2013, 85, 9713-9720.

(65) Liu, H.; Crooks, R. M. J. Am. Chem. Soc. 2011, 133, 17564-17566.

(66) Zang, D.; Ge, L.; Yan, M.; Song, X.; Yu, J. Chem. Commun. 2012, 48, 4683-4685.

(67) Jenkins, S. H.; Heineman, W. R.; Halsall, H. B. Anal. Biochem. 1988, 168, 292-299.

(68) Tang, H. T.; Lunte, C. E.; Halsall, H. B.; Heineman, W. R. Anal. Chim. Acta 1988, 214, $187-195$ 
(69) Bard, A. J.; Faulkner, L. R. Electrochemical Methods, 2nd ed.; John Wiley \& Sons: New York, 2001.

(70) Osteryoung, J.; Odea, J. J. Electroanal. Chem. 1986, 14, 209-308.

(71) Chen, A. C.; Shah, B. Analytical Methods 2013, 5, 2158-2173.

(72) Roche. Elecsys ${ }^{\circledR}$ Troponin $T$ hs, 2013, Retrieved at:

http://www.rochecanada.com/fmfiles/re7234008/package_inserts/TROPONINTHS05092744190-ENGLISH-V4-CAN.pdf.

(73) Yin, Y.; Li, Z.-Y.; Zhong, Z.; Gates, B.; Xia, Y.; Venkateswaran, S. J. Mater. Chem. 2002, $12,522-527$.

(74) Wiseman, M. E.; Frank, C. W. Langmuir 2011, 28, 1765-1774.

(75) Dondorp, A. M.; Desakorn, V.; Pongtavornpinyo, W.; Sahassananda, D.; Silamut, K.;

Chotivanich, K.; Newton, P. N.; Pitisuttithum, P.; Smithyman, A. M.; White, N. J.; Day, N. P. J. PLoS Med. 2005, 2, 1047-1047. 